\section{Multimodal nonlinear optical imaging of atherosclerotic plaque development in myocardial infarction-prone rabbits}

\author{
Alex C. T. Ko, ${ }^{\mathrm{a}, *}$ Andrew Ridsdale, ${ }^{\mathrm{b}}$ Michael S. D. Smith, \\ Leila B. Mostaço-Guidolin, ${ }^{a}$ Mark D. Hewko, ${ }^{a}$ \\ Adrian F. Pegoraro, Elicia K. Kohlenberg, ${ }^{\text {a }}$ \\ Bernie Schattka, ${ }^{a}$ Masashi Shiomi, ${ }^{c}$ Albert Stolow, ${ }^{b}$ and \\ Michael G. Sowa ${ }^{a}$ \\ ${ }^{a}$ National Research Council Canada, Institute for \\ Biodiagnostics, 435 Ellice Avenue, Winnipeg, Manitoba, \\ Canada R3B 1 Y 6 \\ ${ }^{b}$ National Research Council Canada, Steacie Institute for \\ Molecular Sciences, 100 Sussex Drive, Ottawa, \\ Ontario, Canada K1A 0R6 \\ ${ }^{\mathrm{C}}$ Kobe University School of Medicine, Institute of \\ Experimental Animals, 7-5-1, Kusunoki-cho, Chuo-ku, \\ Kobe 650-0017, Japan
}

\begin{abstract}
Label-free imaging of bulk arterial tissue is demonstrated using a multimodal nonlinear optical microscope based on a photonic crystal fiber and a single femtosecond oscillator operating at $800 \mathrm{~nm}$. Colocalized imaging of extracellular elastin fibers, fibrillar collagen, and lipid-rich structures within aortic tissue obtained from atherosclerosis-prone myocardial infarction-prone Watanabe heritable hyperlipidemic (WHHLMI) rabbits is demonstrated through two-photon excited fluorescence, second harmonic generation, and coherent anti-Stokes Raman scattering, respectively. These images are shown to differentiate healthy arterial wall, early atherosclerotic lesions, and advanced plaques. Clear pathological changes are observed in the extracellular matrix of the arterial wall and correlated with progression of atherosclerotic disease as represented by the age of the WHHLMI rabbits. ๑ 2010 Society of Photo-Optical Instrumentation Engineers. [DOI: 10.1117/1.3353960]
\end{abstract}

Keywords: atherosclerosis; nonlinear optical microscopy; photonic crystal fiber; coherent anti-Stokes Raman scattering; two-photon excited fluorescence; second harmonic generation.

Paper 09412LR received Sep. 18, 2009; revised manuscript received Jan. 27, 2010; accepted for publication Feb. 10, 2010; published online Mar. 12, 2010.

Atherosclerosis is the primary cause of heart disease, stroke, and lower limb amputation worldwide. It is a progressive disease characterized by chronic inflammation of injured intima and is associated with fatty plaque deposits in the arteries. ${ }^{1,2}$ Early atherosclerosis cannot be reliably detected by current clinical methods, therefore the disease is often overlooked until at a more advanced stage. The development of new tools that provide greater sensitivity and specificity for early detection and differentiation of atherosclerotic plaques would help our understanding of early disease and help

*Address all correspondence to: Alex C.T. Ko. Tel: 204-984-4622; Fax: 204-9845472; E-mail: alex.ko@nrc-cnrc.gc.ca establish preventative regimens that would slow disease progression.

Recently, nonlinear optical (NLO) microscopy has emerged as a powerful tool for tissue imaging. It is a labelfree method with high sensitivity and specificity for major extracellular molecules. Its optical sectioning capability presents a means of $3-\mathrm{D}$ in vivo imaging that would be useful in the context of atherosclerosis diagnostics. Several studies have demonstrated imaging of arterial tissue using NLO microscopy, ${ }^{3-6}$ including studies imaging atherosclerotic lesions using a multimodal coherent anti-Stokes Raman Scattering (CARS) microscope based on two tightly synchronized Ti:sapphire lasers and a swine animal model. ${ }^{6,7}$ In our study, we demonstrate label-free visualization of the extracellular matrix of arterial lumen and atherosclerotic plaques using a photonic crystal fiber (PCF)-based multimodal NLO microscope employing only a single femtosecond oscillator. Twophoton excited autofluorescence (TPEF) is able to specifically image extracellular elastin fibers, second harmonic generation (SHG), type-1 collagen fibrils, and CARS lipid-rich structure or extracellular lipids droplets in unstained bulk intact tissue, indicating the methods are particularly suited to understanding the role and interplay between these key extracellular molecules involved in plaque development.

PCF-based CARS was recently reported as an alternative CARS imaging method in biology. ${ }^{8,9}$ Because it only requires a single femtosecond laser, PCF-based CARS can be easily integrated into existing multiphoton microscopes with minimal reconfiguration, and provides CARS capability at a relatively low cost. Its portability and its future potential of being developed into an all-fiber, single-laser-based CARS imaging system are better than a conventional dual-color system. From a clinical research point of view, this CARS configuration is better adapted to being implemented in a surgical environment.

Colocalized elastin/type-1 collagen lipid imaging of bulk arterial tissues freshly harvested from WHHLMI rabbits is achieved at micron resolution using a home-built NLO microscope. The TPEF and SHG signals are obtained using the femtosecond pump pulses, with the CARS signal being generated by spatially and temporally overlapping the pump pulse with the Stokes pulse produced in a PCF that is synchronously pumped by the same femtosecond laser. Our goals in this study are to investigate the potential of a PCF-based multimodal NLO imaging system for bulk tissue imaging and for extracting biomorphological information related to atherosclerotic lesion development in myocardial infarction-prone rabbits.

Figure 1 illustrates our PCF-based multimodal NLO imaging system used in this study. A green-pumped Ti:sapphire oscillator (Tsunami, Spectra-Physics, Mountain View, California) is the laser source, and the output wavelength is centered at $800 \mathrm{~nm}$ with a pulse duration of $100 \mathrm{fs}$, an $80-\mathrm{MHz}$ repetition rate, and average output power of $1 \mathrm{~W}$. The femtosecond pulses from the Ti:sapphire oscillator first pass through a Faraday isolator and then a pair of GTI chirp mirrors (Layertec GmbH, Germany). These mirrors are used to compensate for the large group velocity dispersion (GVD) caused by the Faraday isolator. The femtosecond pulses are then split

1083-3668/2010/15(2)/020501/3/\$25.00 @ 2010 SPIE 


\section{JBO Letters}

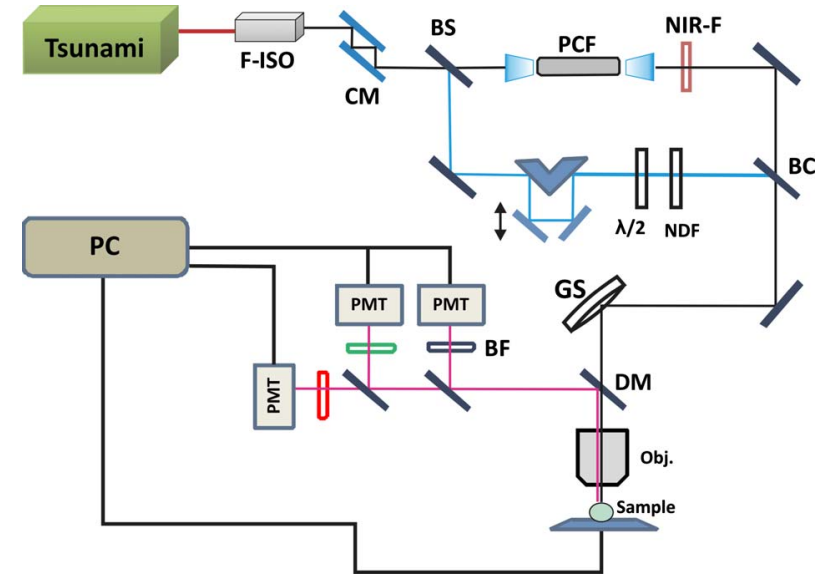

Fig. 1 Schematic of the inhouse-built nonlinear optical laser-scanning microscope with the Stokes pulses being generated in PCF, which is synchronously pumped by the same Ti:sapphire laser that provides the pump pulse. F-ISO: Faraday isolator; CM: chirp laser mirrors; BS: beamsplitter; NIR-F: near-IR filter; BC: beam combiner; GS: galvo scanner; DM: dichroic mirror; OBJ: objective lens; BF: bandpass filter; NDF: neutral density filter; and $\lambda / 2$ : half waveplate.

into two beams at a beamsplitter. The reflected "pump" pulse is transmitted through a series of optical components outlined in Fig. 1, while the transmitted pulse is coupled into a PCF (NL-1.4.775-945, NTK Photonics A/S, Denmark) through a $40 \times$ objective lens to generate a supercontinuum (SC). This SC emission is recollimated through a $20 \times$ objective lens and filtered through a near-infrared (NIR) filter to select only the NIR portion $(950 \mathrm{~nm}<\lambda<1150 \mathrm{~nm})$, which is then used as the Stokes pulses for generating the CARS signal. The pump and Stokes pulses are combined at a beam combiner and sent collinearly into the microscope assembly. Epi-NLO signals are collected through an Olympus $20 \times, 0.75-N A$ objective lens (UPlanSApo) and then detected using nondescanned PMT modules (H-9656, Hamamatsu, Bridgewater, New Jersey). Spectral separation of TPEF, SHG, and CARS signals is achieved through an array of dichroic lenses and bandpass filters. A $40 \times, 0.8-\mathrm{NA}$, water immersion objective lens (Olympus LUMPlanFl/lR) is used to collect tissue images of greater spatial and depth resolution in selected regions of interest. Typically, $25 \mathrm{~mW}$ of pump and $8 \mathrm{~mW}$ of Stokes are used at the sample for imaging. ScanImage software ${ }^{10}$ (Cold Spring Harbor Laboratory, New York) is used for image acquisition and controlling translational stage movement during depth imaging. For images shown, total pixel dwell time is $21 \mu \mathrm{s}$ from an average of four scans. ImageJ ver 1.42b (National Institutes of Health, Bethesda, Maryland) is used for image viewing and post-processing.

Animal studies were approved by the local Animal Care Committee of the National Research Council of Canada. This study uses the myocardial infarction-prone Watanabe heritable hyperlipidemic (WHHLMI) rabbit model ${ }^{11}$ which highly resembles human in lipoprotein metabolism compared to other models, and develops atherosclerotic plaques rapidly due to a hereditary defect in low-density lipoprotein processing. Seven WHHLMI rabbits were used for this study. Six rabbits were each sacrificed at $4,10,11,16,18$, and 22 months, whereas the seventh rabbit died naturally at the age of 24 months. For each rabbit, fresh aorta was dissected from the ascending aorta to the external iliac artery, rinsed in heparinized saline,
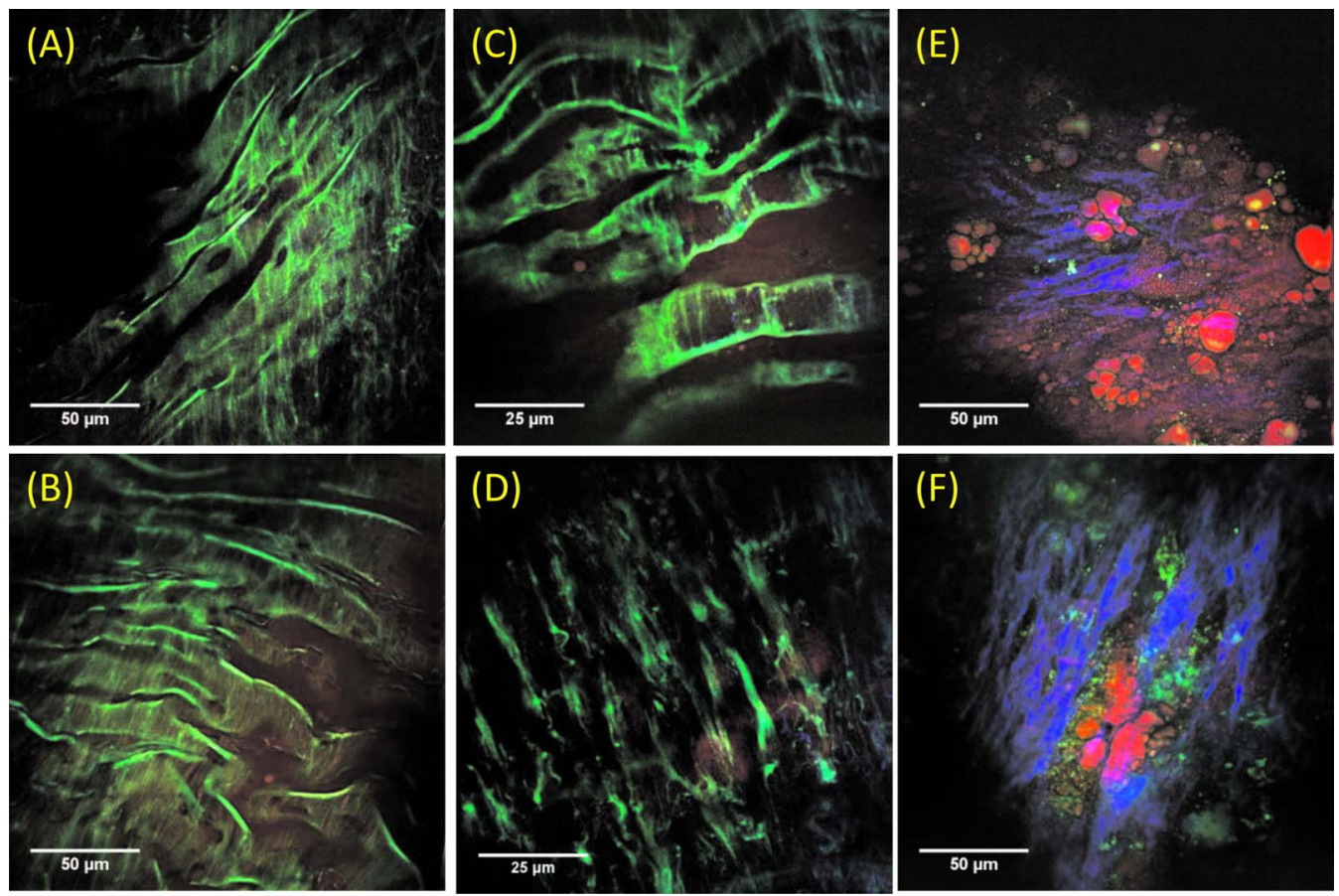

Fig. 2 Representative epi-NLO images collected from healthy arterial lumen on a (a) 4-month-old and (b) 10-month-old WHHLMI rabbit using $20 \times$ air objective lens, and on a 4-month-old rabbit using $40 \times$ WI lens at (c) lumen surface and (d) $\sim 20 \mu \mathrm{m}$ depth from surface. Representative epi-NLO images of arterial lumen surface obtained from (e) an early atherosclerotic lesion and (f) an advanced atherosclerotic lesion using $20 \times$ air lens. Green:TPEF (elastin or other fluorescent particles). Blue:SHG (collagen). Red:CARS (lipid-rich structure). (Color online only.) 

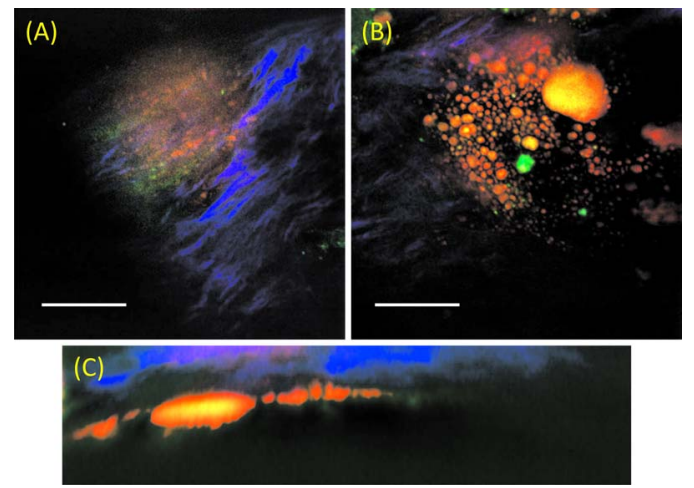

Fig. 3 Epi-NLO images of an advanced plaque obtained at (a) $\sim 10 \mu \mathrm{m}$ depth, (b) $\sim 60 \mu \mathrm{m}$ depth from the lumen surface, and (c) the side-view of the image stack showing a collagen cap overlaying lipid-rich bulk. Blue:SHG (collagen), and red/orange:CARS (lipid-rich structure). The scale bar is $25 \mu \mathrm{m}$. (Color online only.)

and then subdivided into $\sim 20$ - to 30 -mm sections. Each section was cut open longitudinally, exposing the luminal surface. The samples were placed in petri dishes with the luminal surface facing up. Phosphate buffered saline solution was applied to the samples periodically to maintain hydration. Healthier arterial lumen from the 4-month-old rabbit showed mostly smooth and flat surfaces with occasional raised lesions. Plaque-covered luminal surfaces were observed in rabbits aged 10 to 24 months with the burden increasing with age. This observation is consistent with the result reported on progressive atherosclerosis in the WHHLMI rabbits. ${ }^{12}$

Representative epi-NLO images of healthy luminal surfaces measured on 4- and 10-month-old rabbits are shown in Figs. 2(a) and 2(b), respectively. Near the surface of the vessel wall, a layer of membrane structure giving rise to strong TPEF signal (shown in green) is evident. At a depth of approximately $20 \mu \mathrm{m}$, a different fibril structure appears in the TPEF images with the orientation of these structural fibers running nearly perpendicular to that of the membrane layer detected closer to the surface. [Fig. 2(d)]. The membraneshaped structure shown in Figs. 2(a)-2(c) is consistent with internal elastic lamina in arterial tunica intima, and the fibril structure shown in Fig. 2(d) is believed to be the bulk elastin network in arterial tunica media. A representative NLO image acquired from the surface of an early atherosclerotic lesion found on the arterial wall of the 10-month-old rabbit is illustrated in Fig. 2(e). Unlike in the images acquired from healthy luminal surfaces, no internal elastic lamina is visible. Instead, scattered collagen fibers, accumulated lipid-rich structures, and nonfibrous fluorescent structures emerged from the image. Images acquired from advanced plaque reveal lumen pathology similar to those of early lesions, with differences in collagen fibril morphology and denser systems of nonfibrous fluorescent structures. Older, more advanced plaques show thicker and directional collagen fibrils, as seen in Fig. 2(f), whereas early and midstage lesions show thinner and less directional collagen fibrils [Fig. 2(e)]. Figure 2(e) also shows the accumulation of extracellular lipid aggregates and the early formation of collagen fibrils within a lesion.

Depth scanning of an advanced plaque on a 22-month-old rabbit artery shows a thin collagen fibril layer overlaying a pool of lipid debris, a typical pathology of a rupture-prone atherosclerotic plaque. Figures 3(a) and 3(b) are two focal depth images obtained from the same location on an advanced plaque at depths approximately 10 and $60 \mu \mathrm{m}$ respectively from the lumen surface. In Fig. 3(c) a reconstructed side-view image representing a depth profile of this particular plaque shows a collagen layer on top of a lipid-rich pool.

In conclusion, we demonstrated multimodal nonlinear optical imaging of bulk arterial tissue from WHHLMI rabbits using a cost-effective PCF-based nonlinear optical microscope. Epi-NLO imaging of unsectioned arteries allowed label-free visualization of extracellular components relevant to the development of atherosclerosis. Clear differences in the surface biochemical morphology were observed between healthy artery wall, early atherosclerotic lesions, and advanced plaques. In particular, differences in collagen fibril structures were noted between early and advanced lesions. Such changes in collagen morphology detected by SHG may provide a clinical measure for differentiating plaque burden.

\section{Acknowledgment}

The authors acknowledge Vijay Iyer for the assistance provided in using ScanImage, and Jeri Friesen for her assistance in preparing figures. This work was supported by the National Research Council Canada, Genomics and Health Initiative.

\section{References}

1. P. Libby, "Atherosclerosis: disease biology affecting the coronary vasculature," Am. J. Cardiol. 98[suppl], 3Q-9Q (2006).

2. G. K. Hansoon, "Inflammation, atherosclerosis, and coronary artery disease," N. Engl. J. Med. 352, 1685-1695 (2005).

3. A. Zoumi, X. Lu, G. S. Kassab, and B. J. Tromberg, "Imaging coronary artery microstructure using second-harmonic and two-photon fluorescence microscopy," Biophys. J. 87(4), 2778-2786 (2004).

4. M. B. Lilledahl, O. A. Haugen, C. D. L. Davis, and L. O. Svaasand, "Characterization of vulnerable plaques by multiphoton microscopy," J. Biomed. Opt. 12(4), 044005 (2007).

5. T. T. Le, I. M. Langohr, M. J. Locker, M. Sturek, and J. X. Cheng, "Label-free molecular imaging of atherosclerotic lesions using multimodal nonlinear optical microscopy," J. Biomed. Opt. 12(5), 054007 (2007).

6. H. W. Wang, T. T. Le, and J. X. Cheng, "Label-free imaging of arterial cells and extracellular matrix using a multimodal CARS microscope," Opt. Commun. 281, 1813-1822 (2008).

7. H. W. Wang, I. M. Langohr, M. Sturek, and J. X. Cheng, "Imaging and quantitative analysis of atherosclerotic lesions by CARS-based multimodal nonlinear optical microscopy," Arterioscler., Thromb., Vasc. Biol. 29, 1342-1348 (2009).

8. A. F. Pegoraro, A. Ridsdale, D. J. Moffatt, Y. Jia, J. P. Pezacki, and A. Stolow, "Optimally chirped multimodal CARS microscopy based on a single Ti:sapphire oscillator," Opt. Express 17(4), 2984-2996 (2009).

9. S. Murugkar, C. Brideau, A. Ridsdale, M. Naji, P. K. Stys, and H. Anis, "Coherent anti-Stokes Raman scattering microscopy using photonic crystal fiber with two closely lying zero dispersion wavelengths," Opt. Express 15(21), 14028-14037 (2007).

10. T. A. Pologruto, B. L. Sabatini, and K. Svoboda, "ScanImage: flexible software for operating laser scanning microscopes," Biomed. Eng. Online 2, 13 (2003).

11. M. Shiomi, T. Ito, S. Yamada, S. Kawashima, and J. Fan, "Development of an animal model for spontaneous myocardial infarction (WHHLMI rabbit)," Arterioscler., Thromb., Vasc. Biol. 23, 1239 (2003).

12. T. Itp, S. Yamada, and M. Shiomi, "Progression of coronary atherosclerosis relates to the onset myocardial infarction in an animal model of spontaneous myocardial infarction (WHHLMI rabbits)," Exp. Anim. 53, 339-346 (2004). 